\title{
The dissatisfied total knee arthroplasty patient. New technologies-the white knight in shining armor coming to their rescue?
}

\author{
E. Thienpont ${ }^{1} \cdot$ A. Klasan ${ }^{2}$ \\ Accepted: 8 October 2021 / Published online: 16 October 2021 \\ (c) The Author(s), under exclusive licence to Springer-Verlag GmbH Germany, part of Springer Nature 2021
}

Knee osteoarthritis $(\mathrm{OA})$ and its prosthetic treatment went through a major evolution over the last 40 years since its inception. From a procedure, where patients signed initially two consent forms; one for total knee arthroplasty (TKA) followed by a plaster cast immobilization and a second for a mobilization under anesthesia (MUA) a few weeks later to start bending again, TKA became today a procedure performed in ambulatory surgery centers (ASC) with patients walking out on the same day [1]. The drive behind all of this has always been, from the start, the wish of surgeons to obtain the best possible results for their patients after joint replacement. This ambition became clear when the "Forgotten Joint Score" was developed [2]. A knee-specific score, evaluating in a very detailed way, how aware patients are about their arthritic knee before [3] and after knee- and hip arthroplasty [2, 4]. Today, still around 20 percent of TKA patients are dissatisfied with their procedure, substantially more than after hip replacement. Orthopedic surgeons and implant producers often wish to reduce this dissatisfaction to a surgically solvable problem and therefore limited to a purely mechanical cause. They might neglect well-known issues such as the preoperative absence of bone on bone OA, pre-existing risk factors for acute and chronic pain, chronic morphine use, central pain sensitization, inflammatory neuropathy around the knee, pain catastrophizing, referred pain, workman compensation and many other psychosocial factors $[5,6]$.

Antonio Klasan is an editorial board member of Archives of Orthopaedic and Trauma Surgery and is an associate editor for BMC Musculoskeletal Disorders.

E. Thienpont

emmanuel.thienpont@uclouvain.be

1 Cliniques Universitaires Saint Luc, Av. Hippocrate 10, 1200 Brussels, Belgium

2 Johannes Kepler University Linz, Altenbergerstraße 69, 4040 Linz, Austria
For surgeons and the orthopedic industry, these unsatisfied patients remain the reason for their continuous quest to do better by research and development, leading to different innovations trying to solve these problems. In the past two decades, the TKA patient' dissatisfaction was explained by sizing issues leading to overhang and pain or downsizing and flexion instability. This led to the development of many different sizes with more representative anatomical aspect ratios and better surface matching. The next attempt to solve dissatisfaction came with the introduction of more partial knee replacements, where resurfacing of only the diseased side of the knee would lead to better results. Indications, expertise with surgical technique, the balance between quicker recovery and a more natural feeling of the knee versus a threefold higher revision rate seem to be limiting factors to convince all surgeons in favor of unicompartmental knee arthroplasty (UKA). Furthermore, a maximum $50 \%$ of all patients might be treated with UKA, including statistics from high volume centers. And now lately, we are observing a trend towards personalized alignment. Each human being has its own unique type of alignment, developed during his lifetime and approaching this native alignment more closely, would lead to a better outcome. The ambition of surgeons to start implanting knee arthroplasties in their more oblique native joint line position is not new and as any re-invention of an old failed philosophy, comes with a new engineered technological solution, such as precision-enabling robots today. Hungerford and Krackow developed this oblique alignment philosophy 40 years ago as anatomical alignment (AA), with a fixed $3^{\circ}$ femoral valgus and $3^{\circ}$ tibial varus in the coronal plane and a femoral component aligned parallel to the posterior condylar axis (PCA) in the axial plane. They observed unfortunately alignment outliers due to the limitations of their simple instrumentation and failure of the Porous Coated Anatomic-implant [7]. As a reaction, mechanical alignment (MA) as proposed by Insall, became the gold standard to equally load the polyethylene on both sides and to avoid important component position outliers 
[1]. Both these two-dimensional, systematic alignment techniques, are now considered a potential cause for pain and dissatisfaction after TKA [8]. As a reaction, a three-dimensional and patient-specific alignment strategy was proposed by Howell, instrumented with patient-specific instruments (PSI) [9]. Precision-enabling technology, such as computernavigation and patient-specific instruments, used for a decade to avoid these supposedly dangerous surgical outliers from the neutral mechanical axis $[10,11]$, would now help us to implant the same prostheses in different outlier positions, depending on the surgeon's preferred alignment philosophy. Because of this drastic change in the knee arthroplasty world, we urge a consensus position on the different alignment philosophies. Lustig et al. performed an excellent review in 2021, but with some remaining uncertainties or assumptions about the different "hybrid" alignment options [12-14]. Therefore, we contacted Matthew Abdel, Johan Bellemans, Stephen Howell, Sebastien Parratte, Charles Rivière, Philip Winnock de Grave and Simon Young and came thanks to their help to the following conclusions:

Kinematic alignment (KA) wants to co-align the axes of the femoral and tibial components with the three "kinematic" axes of the native or pre-arthritic knee, adapting the implant's position to its native anatomy and soft tissue envelope [9]. However, surgeons started modifying this alignment philosophy to their own believes and convictions, leading to other KA terminologies.

Restricted Kinematic Alignment (rKA) wants to reproduce the patient's constitutional knee anatomy with KA, but within a safe range avoiding extreme pathological anatomies. Coronal alignment of the femoral and tibial component is limited within $\pm 5^{\circ}$ of neutral. The overall combined lower limb coronal alignment must be within $\pm 3^{\circ}$ of neutral HKA. Priority is given to preserve the femoral anatomy and to adapt the gaps with the varus/valgus position of the tibia within the given safe range [13].

Inverse Kinematic Alignment (iKA) aims at resurfacing the tibia with equal medial and lateral resections corresponding to implant thicknesses while maintaining the native tibial joint line obliquity. Priority is given to preserve the tibial anatomy and the extension gap is balanced by adjusting the femoral resection parallel to the tibial cut. This is a tibia-first technique with balanced flexion-extension gaps [14].

Adjusted Mechanical Alignment (aMA) adapts neutral mechanical alignment $\left(180^{\circ}\right)$ to obtain slight undercorrection in the coronal plane, often leaving some constitutional varus since an important segment of the population might have a native HKA of $178^{\circ}$ [15]. This undercorrection is usually obtained by a distal femoral cut of $5^{\circ}$ (with an AMA of $7^{\circ}$ ) leading to a slight varus position of the femoral component and reducing the need for medial soft tissue releases. For the valgus knee, the same distal femoral valgus cut of $5^{\circ}$ would retain some valgus at the femoral side (with an AMA of $3^{\circ}$ ). The rotational alignment follows the epicondylar axis on the femur and Baldini's line on the tibial side [16].

Functional Alignment (FA): a precision-enabled surgical technique allowing pre-or intra-operative assessment of resection thicknesses, joint gaps and component alignment. This technique combines computer-based simulated calculations combining measured resection and gap-balancing techniques. The gaps are balanced beginning with an anatomical start point of the femur by changing component targets in all three planes within the limits of the safe zone of neutral mechanical axes (femur, tibia, HKA) and the surgical epicondylar axis [17]. The choices are mechanical/anatomical femoral with tibial functional alignment and mechanical/anatomical tibial alignment with femoral functional alignment.

All these different alignment philosophies, except kinematic alignment, remain within the boundaries of a neutral mechanical axis $\left(180^{\circ}\right)$ with an extension of the classic safe zone $\left( \pm 3^{\circ}\right)$ with an additional $3^{\circ}$. The only remaining decision is to perform the adaptive corrections on the femur or on the tibia and whether to perform it as a femur-first (KA/ rKA) or tibia-first technique (iKA) [9, 13, 14].

\section{Femur driven}

\section{Coronal options}

Mechanical alignment: distal cut perpendicular to the femoral mechanical axis or undercorrection $\left(5^{\circ}\right.$ valgus AMA) with adjusted technique.

Anatomical alignment: distal cut at fixed $3^{\circ}$ valgus of femoral mechanical axis.

Kinematical alignment: distal cut at a variable angle to the femoral mechanical axis depending on the individual anatomic variability, aiming to resect the exact amounts of bone and cartilage, medially and laterally, to match the implant thickness-unrestricted or restricted (max $5^{\circ}$ valgus) or inverse (parallel to tibia).

Functional alignment: distal cut anatomical either mechanical with extension gap balancing leading to a functional tibial cut respecting the soft tissue sleeve to allow volumetric implantation without releases. Limits are $3^{\circ}$ varus and $6^{\circ}$ valgus.

\section{Axial options}

Mechanical alignment: $3^{\circ}$ external rotation referenced of the posterior condylar axis of femur to align the implant with the Surgical Epicondylar Axis (SEA).

Anatomical alignment: neutral rotation following the posterior condylar axis of femur. 
Kinematical alignment: neutral rotation following the posterior condylar axis of femur

and respecting the volumetric need of the implant to reconstruct the individual posterior condylar offsets. In inverse KA, the femoral rotation is guided by the collaterals as a gap balancing technique with a tensioner (on average $2^{\circ}$ of external rotation).

Functional alignment: neutral rotation following the posterior condylar axis of femur, but with rotational freedom to allow volumetric replacement without releases. The limits are $6^{\circ}$ internal to $6^{\circ}$ external rotation.

\section{Sagittal options}

All philosophies follow the native femoral flexion of about $3^{\circ}$, but without excessive flexion of more than $5^{\circ}$ because of the trochlea and proximal patellar instability.

\section{Tibia driven}

\section{Coronal options}

Mechanical alignment: proximal cut perpendicular to the tibial mechanical axis - also for adjusted mechanical alignment.

Anatomical alignment: proximal cut at a fixed $3^{\circ}$ of varus from the tibial mechanical axis.

Kinematical alignment: proximal cut at a variable angle to the tibial mechanical axis depending on the individual anatomic variability- unrestricted or restricted $\left(\max 5^{\circ}\right)$. For inverse kinematical alignment tibial cut parallel to the native oblique tibial joint line (tide mark area) replacing the same amounts of bone on both sides.

Functional alignment: mechanical or anatomical tibial cut for tibia-driven technique with soft-tissue extension and flexion gap balancing for femoral side allowing volumetric substitution without release. The limits are $6^{\circ}$ varus and $3^{\circ}$ valgus.

\section{Axial options}

Mechanical alignment: medial 1/3 of the tibial tubercle.

Adjusted mechanical alignment: Baldini's line following the anterior anatomy of the tibia.

Anatomical alignment: not available.

Kinematical alignment: along the longitudinal axis of the lateral plateau for unrestricted and restricted KA. For inverse $\mathrm{KA}$, line from the medial border of the patellar tendon to the middle of the insertion of the posterior cruciate ligament (Akagi's Line) [18].
Functional alignment: medial border of the patellar tendon to the middle of the insertion of the posterior cruciate ligament (Akagi's line) [18].

\section{Slope options}

All techniques follow the native medial slope (limited to $5^{\circ}$ ) with a maximal combined angle of femoral flexion and posterior slope of $8^{\circ}$.

At the cradle of these discussions were the observations from Parratte et al. that the long-term follow-up of implants, outside of the safe zone of HKA $180^{\circ} \pm 3^{\circ}$, were not presenting with worse survivorship than those TKAs neutrally aligned [19]. However, in that study the authors didn't analyse the individual components' position, but the overall alignment of the limb. Bellemans et al. [15] published that a segment of the normal population presents with a native varus alignment or constitutional varus and may not need necessarily perfect postoperative neutral mechanical axes. The above findings led to the concept of varus and valgus bone morphotypes by Thienpont et al. who showed that varus and valgus deformity have characteristics of their own typical deformity outside of the worn joint. This starts at the hip (varus or valgus neck), goes to the diaphysis of femur and tibia and ends in the load-bearing position of the foot [20]. The authors of this editorial believe it is time to understand coronal deformity in a more profound way as Hirschmann et al. and MacDessi et al. did with their extensive coronal alignment classifications [21, 22].

HKA-measured coronal alignment can be a result of epiphyseal or metaphyseal wear (intra-articular) and the concomitant soft tissue laxity of the opposite convex side. But HKA measured deformity, can also have a contribution from the specific bony anatomy at the epiphyseal, metaphyseal and diaphyseal level [23, 24]. The impact of the extra-articular anatomy should be analysed before deciding in what type of obliquity the implant can be positioned. The native anatomy of the epiphyseal joint line of the knee is usually medially sloped [25]. Since mostly symmetrical implants are used to reconstruct asymmetrical gaps, this type of implants need to be positioned obliquely to respect the native gaps better. All the above-mentioned types of alignment try to solve a volumetric problem of creating enough space for the implant without additional soft tissue balancing. Today it is still based on mathematical calculations of alignment corrections and measured resections, but few have data about articular pressures obtained with sensors. In the short term, less soft tissue damage will of course lead to a better outcome and faster rehabilitation, but does it also lead to better knee kinematics? Are all these mathematical calculations not only possible because the resection of the anterior and posterior cruciate ligaments alters the gap kinematics? Surgeons performing measured resection know 
very well that most resections are very variable, despite that spacers or feeler gauges show adequate space for the metal components.

If the measured deformity has only an intra-articular origin, all the above resurfacing techniques will correct the alignment to $\pm 3^{\circ}$ neutral alignment. If the deformity is also extra-articular, a reduction of the alignment outlier can only be obtained by a correction within the joint. TKA should be recognized as an intra-articular osteotomy of the lower limb combined with a resurfacing of the epiphyseal anatomy. Has it been analysed what the difference in joint line obliquity would be for an individual patient, if treated with mechanical, anatomical or kinematical alignment and what the impact on the overall HKA alignment would be for these different strategies? Is there a clinical difference between the kinematic functioning of the knee with anatomical alignment versus kinematical? Is what we call kinematic, not simply patient specific? And is functional alignment, not just anatomical within the safe zone of mechanical alignment?

This entire discussion about coronal deformity and the different ways to reconstruct the native joint line with its natural obliquity, can be reduced to the observation that there are different offsets in the human knee. But is respecting only the coronal joint obliquity enough to observe better clinical outcome? Maybe we should not only consider the distal offset of the femur but also its posterior condylar offset and the anterior offset of the trochlea. Each condyle has furthermore its own unique $\mathrm{J}$-curve with its own radius of curvature determinating the potential for medial collateral ligament isometry and lateral condylar roll back around this medial pivot point. At the tibial level, it is more about volumetric replacement and adequate posterior slope to allow the collaterals to function in the absence of the central pivot. In general, with conventional TKA and especially with mechanically aligned TKAs, the lateral side of the human knee is distalized and posteriorized. This lateral overstuffing, both in flexion and extension, is probably well tolerated in those that have a looser lateral side and less in the others. A patient-specific knee, designed according to the unique anatomic features of each patient, could potentially make a difference. This more resurfacing type of implant, with respect for the individual J-curves and different offsets allows standard mechanical cuts since the anatomic individuality is reconstructed at the epiphyseal level. These implants unfortunately still come at a higher price, without proven superiority, but not inferior to off-the-shelf knees either. The new alignment concepts in vogue today, asking for precisionenabling technologies, change the actual cost calculation. Surgeons need to add now the variable cost of robotic hardware (lease price robot/amount of cases), robot disposables, technical assistance, maintenance and more surgical time to the real cost of their off-the-shelf implant, bringing the bottom line closer to the cost of a patient-specific implant. It's time for the patient-specific implant producers to come out of their niche corner and become real competitors for the big four. The door is wide open now!

However, we must remain critical and try to keep a helicopter view on this new trend of robotic surgery, where navigation is combined with soft tissue gap modeling. What has it brought us new since the era of navigation?

Have we finally decided on what the right alignment target should be, so we can start talking about improved accuracy and not only about precision?

Have we finally decided what amount of soft tissue laxity we need in our collateral ligaments in flexion and extension and what difference in laxity between the medial and lateral side after prosthetic replacement? The same laxity in cruciate retaining (CR) and posterior-stabilized (PS) designs?

How can we know if osteoporotic bone supports overload of a metallic implant on the undercorrected side and what about putting a tibial component in $3^{\circ}$ of varus in a valgus morphotype patient, who has been loading his lateral bone for years? The medial side will be osteoporotic according to Wolf's law.

In the face of the Thienpont and Parvizi varus classification [23] and the Krackow valgus classification [26], how will we handle uncorrectable deformities without soft tissue releases? How much residual coronal deformity can be tolerated cosmetically and still obtain happy postoperative patients? How accurate do we need to be with hybrid/ patient-specific alignment to reduce the $20 \%$ of unsatisfied patients treated with systematic alignment.

Total knee arthroplasty is a complex procedure because joint replacement consists of a volumetric substitution with metallic and plastic components of native tissues, such as cartilage and meniscus, which will always have another elasticity modulus [27]. Furthermore, surgeons try to reconstruct anatomical joint line obliquity, but in the partial (CR) or complete absence (PS) of the central pivot ligaments. Can we just expect that the anatomical coronal plane reconstruction will solve all our problems? Did we ever wonder why this discussion is not actual for unicompartmental or revision knee arthroplasty [28]?

This volume on new technologies shows how passionate knee surgeons are about their job and the best outcome they want to give to their patients. The special issue tries to give the necessary information to surgeons thinking about selecting an assistive technology to improve the outcome of their patients. A wide overview of available technologies is offered with conceptual papers and clinical papers, from unicompartmental knee arthroplasty to revision knee arthroplasty. These authors are proud that their colleagues are thinking about alignment concepts and working so hard for their patients. If the new technologies are not the white knights, knee surgeons will be. 
Acknowledgements We want to thank Matthew Abdel, Johan Bellemans, Stephen Howell, Sebastien Parratte, Charles Rivière, Philip Winnock de Grave and Simon Young for their email discussions about the different types of alignment they advocate. A special thank you goes to Thomas Heyse for his arbitrary role as an editor during our difficult moments of choice.

\section{Declarations}

Conflict of interest Emmanuel Thienpont declares as financial interest that he received royalties from Zimmer Biomet and Medacta and has a design agreement with Conformis and Lima. He performs consultancy and advisory work for Conformis, Convatec, Fidia, Lima and Zimmer Biomet. He receives institutional support from Zimmer Biomet. As a non-financial interest, he declares to be board member of the European Knee Society.

\section{References}

1. Insall J, Scott WN, Ranawat CS (1979) The total condylar knee prosthesis. A report of two hundred and twenty cases. J Bone Joint Surg Am 61:173-180

2. Behrend H, Giesinger K, Giesinger JM, Kuster MS (2012) The "forgotten joint" as the ultimate goal in joint arthroplasty: validation of a new patient-reported outcome measure. J Arthroplasty $27: 430-436$

3. Thienpont E, Vanden Berghe A, Schwab PE, Forthomme JP, Cornu O (2016) Joint awareness in osteoarthritis of the hip and knee evaluated with the "Forgotten Joint" score before and after joint replacement. Knee Surg Sports Traumat Arthrosc 24:33446-43351

4. Thienpont E, Opsomer G, Konninckx A, Houssiau F (2014) Joint awareness in different types of knee arthroplasty evaluated with the Forgotten Joint Score. J Arthroplasty 29:48-51

5. Grosu I, Lavand'homme P, Thienpont E (2014) Pain after knee arthroplasty: an unresolved issue. Knee Surg Sports Traumatol Arthrosc 22:1744-1758

6. Deumens R, Grosu I, Thienpont E (2015) Surgically induced neuropathic pain: understanding the perioperative process. Ann Surg 261:e161-162

7. Hungerford DS, Kenna RV, Krackow KA (1982) The porouscoated anatomic total knee. Orthop Clin North Am 13:103-122

8. Cherian JJ, Kapadia BH, Banerjee S, Jauregui JJ, Issa K, Mont MA (2014) Mechanical, anatomical and kinematic axis in TKA: concepts and practical applications. Curr Rev Musculoskelet Med 7:89-95

9. Howell SM, Howell SJ, Kuznik KT, Cohen J, Hull ML (2013) Does a kinematically aligned total knee arthroplasty restore function without failure regardless of alignment category? Clin Orthop Relat Res 471(3):1000-1007

10. Thienpont E, Fennema P, Price A (2013) Can technology improve alignment during knee arthroplasty. Knee 20(Suppl 1):21-28

11. Thienpont E, Schwab PE, Fennema P (2017) Efficacy of patientspecific instruments in total knee arthroplasty: a systematic review and meta-analysis. J Bone Joint Surg Am 99:521-530

12. Lustig S, Sappey-Marinier E, Fary C, Servien E, Parratte S, Batailler C (2021) Personalized alignment in total knee arthroplasty: current concepts. SICOT-J 7(19):1-9

13. Almaawi AM, Hutt JRN, Masse V, Lavigne M, Vendittoli PA (2017) The impact of mechanical and restricted kinematic alignment on knee anatomy in total knee arthroplasty. J Arthroplasty $32: 2133-2140$

14. Winnock de Grave P, Luyckx T, Claeys K, Tampere T, Keller J, Müller J, Gunst P (2020) Higher satisfaction after total knee arthroplasty using restricted inverse kinematic alignment compared to adjusted mechanical alignment. Knee Surg Sports Traumatol Arthrosc. https://doi.org/10.1007/s00167-020-06165-4

15. Bellemans J, Colyn W, Vandenneucker H, Victor J (2012) The Chitranjan Ranawat Award: is neutral mechanical alignment normal for all patients? The concept of constitutional varus. Clin Orthop Relat Res 470(1):45-53

16. Baldini A, Indelli PF, De Luca L, Mariani PC, Marcucci M (2014) Rotational alignment of the tibial component in total knee arthroplasty: the anterior tibial cortex is a reliable landmark. Joints $1: 155-160$

17. Oussedik S, Abdel MP, Victor J, Pagnano MW, Haddad FS (2020) Alignment in total knee arthroplasty. What's in a name? Bone Joint J 102:276-279

18. Akagi M, Oh M, Nonaka T, Harutoshi T, Asano T, Hamanishi C (2004) An anteroposterior axis of the tibia for total knee arthroplasty. Clin Orthop Relat Res 420:213-219

19. Parratte S, Pagnano MW, Trousdale RT, Berry DJ (2010) Effect of postoperative mechanical axis alignment on the fifteen-year survival of modern, cemented total knee replacements. J Bone Joint Surg Am 92:2143-2149

20. Thienpont E, Schwab PE, Cornu O, Bellemans J, Victor J (2017) Bone morphotypes of the varus and valgus knee. Arch Orthop Trauma Surg 137:393-400

21. Hirschmann MT, Moser LB, Amsler F, Behrend H, Leclerq V, Hess S (2019) Functional knee phenotypes: a novel classification for phenotyping the coronal lower limb alignment based on the native alignment in young non-osteoarthritic patients. Knee Surg Sports Traumatol Arthrosc 27:1394-1402

22. MacDessi SJ, Griffiths-Jones W, Harris IA, Bellemans J, Chen DB (2021) Coronal plane alignment of the knee (CPAK) classification. Bone Joint J 103:329-337

23. Thienpont E, Parvizi J (2016) A new classification for the varus knee. J Arthroplasty 31:2156-2160

24. De Muylder J, Victor J, Cornu O, Kaminski L, Thienpont E (2015) Total knee arthroplasty in patients with substantial deformities using primary knee components. Knee Surg Sports Traumatol Arthrosc 23:3653-3659

25. Meier M, Janssen D, Koeck FX, Thienpont E, Beckmann J, Best R (2021) Variations in medial and lateral slope and medial proximal tibial angle. Knee Surgery Sports Traumat Arthrosc 29:939-946

26. Krackow KA, Jones MM, Teeny SM, Hungerford DS (1991) Primary total knee arthroplasty in patients with fixed valgus deformity. Clin Orthop Relat Res 273:9-18

27. Innocenti B, Pianigiani S, Ramundo G, Thienpont E (2016) Biomechanical effects of different varus and valgus alignments in medial unicompartmental knee arthroplasty. J Arthroplasty 31:2685-2691

28. Thienpont E, Cornu O, Bellemans J, Victor J (2015) Current opinions about coronal plane alignment in total knee arthroplasty: a survey article. Acta Orthop Belg 81:471-477

Publisher's Note Springer Nature remains neutral with regard to jurisdictional claims in published maps and institutional affiliations. 